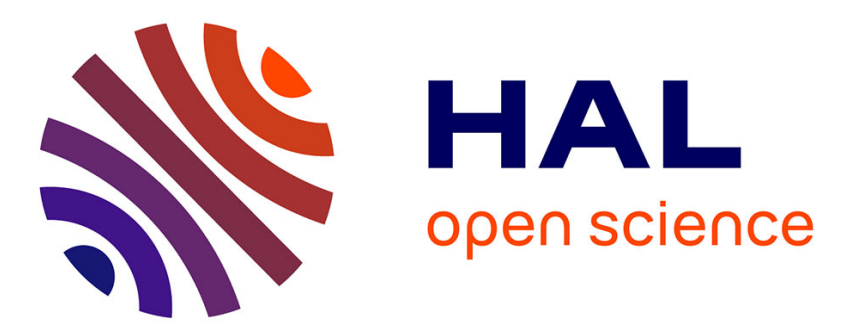

\title{
A set-membership approach to find and track multiple targets using a fleet of UAVs
}

Sébastien Reynaud, Michel Kieffer, Helene Piet-Lahanier, Léon Reboul

\section{To cite this version:}

Sébastien Reynaud, Michel Kieffer, Helene Piet-Lahanier, Léon Reboul. A set-membership approach to find and track multiple targets using a fleet of UAVs. 57th IEEE Conference on Decision and Control (CDC 2018), Dec 2018, Miami, FL, United States. 10.1109/cdc.2018.8619672 . hal-01994781

\section{HAL Id: hal-01994781 \\ https://hal.science/hal-01994781}

Submitted on 25 Jan 2019

HAL is a multi-disciplinary open access archive for the deposit and dissemination of scientific research documents, whether they are published or not. The documents may come from teaching and research institutions in France or abroad, or from public or private research centers.
L'archive ouverte pluridisciplinaire HAL, est destinée au dépôt et à la diffusion de documents scientifiques de niveau recherche, publiés ou non, émanant des établissements d'enseignement et de recherche français ou étrangers, des laboratoires publics ou privés. 


\title{
A set-membership approach to find and track multiple targets using a fleet of UAVs
}

\author{
Sébastien Reynaud, Michel Kieffer, Hélène Piet-Lahanier, and Léon Reboul
}

\begin{abstract}
This paper presents a set-membership approach for the coordinated control of a fleet of UAVs aiming to search and track an a priori unknown number of targets spread over some delimited geographical area. The originality of the approach lies in the description of the perturbations and measurement uncertainties via bounded sets. A set-membership approach is used to address the localization and tracking problem. At each time step, sets guaranteed to contain the actual state of already localized targets are provided. A set containing the states of targets still to be discovered is also evaluated. These sets are then used to evaluate the control input to apply to the UAVs so as to minimize the estimation uncertainty at the next time step. Simulations considering several UAVs show that the proposed set-membership estimator and the associated control input optimization are able to provide good localization and tracking performance for multiple targets.
\end{abstract}

\section{INTRODUCTION}

The search for moving targets of interest over a potentially large area is a challenging topic that can be of use in many real-life applications. These usually time-critical missions may be more efficiently addressed using cooperating agents. Networks of UAVs have already demonstrated their flexibility to mission requirements, robustness to faults, and ability to achieve the global objective in a shorter time.

In this paper, we consider the search and tracking of an a priori unknown number of targets using a fleet of UAVs, with a decision process that may be distributed among the vehicles. Contributions to such problems have been presented in two recent surveys [1], [2]. As described in [1], the initial problem is often split into target detection and target tracking.

Target detection is mostly addressed as a probabilistic information gathering. In [3], the search space is sampled. A Bayesian filter is used to update the probability associated to each point of the sampling grid, and a binary tree search is used to allocate the UAVs to sub-regions of the search area. This approach proves efficient even when the targets outnumber the UAVs but requires to select adequately an $a$ priori subdivision of the search area. A search pattern using Lloyd's algorithm, combined with a Probability Hypothesis Density (PHD) filter to evaluate the occurrence of a target is presented in [4] to achieve distributed coverage and target tracking when the targets may enter or leave the search area.

S. Reynaud, H. Piet-Lahanier, and L. Reboul are with ONERA, 8 Chemin de la Hunière, 91120 Palaiseau, France first_name. last_namedonera.fr

M. Kieffer is with L2S - Univ Paris-Sud, CNRS, CentraleSupelec, Univ Paris-Saclay, 3 rue Joliot-Curie, 91192 Gif-sur-Yvette, France michel.kiefferdl2s.centralesupelec.fr
In the considered scenario, target tracking is performed combining information from multiple sensors. Since they are mobile and their potential locations are constrained by the ability of the carrying platforms, the tracking problem can be formulated as a path planning problem to determine the future UAV sensor locations that are reachable and decrease the target state estimation uncertainty. Various methods have been developed in this context, see, e.g., [5], [6], [7].

In all of the aforementioned approaches, a stochastic framework is used to describe the measurement errors and model uncertainties. As pointed out in [8], the resulting performance may prove sensitive to the a priori assumptions on the probability density functions (pdfs) describing the process and measurement noises. As the definition of suitable pdfs might prove tedious, a set-membership descriptions of uncertainties is suggested in [8]. The only assumption made on the noises and uncertainties is that their realizations remain within known bounds. Using this description, one no longer searches for a single point estimate associated with $a$ posterior density function but for sets guaranteed to contain the target states at each time step.

In this paper, we assume that each UAV is equipped with a sensor able to detect and localize targets in some compact subset of the search area. A distributed set-membership estimator is presented that accounts for information provided by each UAV, but also for the absence of detected target in the sensed subset. A control input for each UAV is derived by predicting the impact of future measurements on the set estimates of target states, and choosing the control which minimizes the estimation uncertainty. To the best of our knowledge, it is the first proposal, considering bounded disturbances, that provides estimates consisting of compact sets guaranteed to contains the true target states.

The paper is organized as follows. Section II describes the multi-target multi-UAV localization problem. Section III presents a distributed estimator that recursively provides sets that are guaranteed to contain the true target positions. Section IV presents a criterion to evaluate the impact of future measurements on target state estimates and a control input design scheme to drive each UAV so as to minimize an upper bound of the estimation uncertainty. In Section V, the set-membership estimator and the control input design algorithms are evaluated on simulations. Section VI concludes this paper.

\section{Problem Formulation}

Consider a fleet of $N_{\mathrm{U}}$ identical UAVs which aim is to search and track $N_{\mathrm{T}}$ potentially moving targets. $N_{\mathrm{T}}$ is fixed 
but not known a priori. Time is sampled with a constant period $T$. At time $k$, (time instant $t=k T$ ), let $\mathbf{r}_{i, k} \in \mathbb{R}^{n_{\mathrm{r}}}$ be the state vector of UAV $i$ and $\mathbf{z}_{j, k} \in \mathbb{R}^{n_{\mathrm{z}}}$ the state vector of Target $j$. Their evolutions with time are modeled as

$$
\begin{aligned}
\mathbf{r}_{i, k+1} & =\mathbf{f}_{k}^{\mathrm{R}}\left(\mathbf{r}_{i, k}, \mathbf{u}_{i, k}\right) \\
\mathbf{z}_{j, k+1} & =\mathbf{f}_{k}^{\mathrm{Z}}\left(\mathbf{z}_{j, k}, \mathbf{v}_{j, k}\right),
\end{aligned}
$$

where $\mathbf{u}_{i, k}$ is the control input for $\mathrm{UAV} i$, to be chosen in a set $\mathbb{U}$ of admissible control inputs; $\mathbf{v}_{j, k}$ are unknown target state perturbations belonging to the known box $\left[\mathbf{v}_{k}\right]$. At time $k=0$, all $\mathbf{z}_{j, 0}$ are assumed to belongto some $a$ priori known compact set $\mathbb{Z}_{0} \subset \mathbb{R}^{n_{\mathrm{z}}}$. The state vectors of the UAV fleet and of the targets are $\mathbf{r}_{k}^{\mathrm{T}}=\left(\mathbf{r}_{1, k}^{\mathrm{T}}, \ldots, \mathbf{r}_{N_{\mathrm{U}}, k}^{\mathrm{T}}\right)$ and $\mathbf{z}_{k}^{\mathrm{T}}=\left(\mathbf{z}_{1, k}^{\mathrm{T}}, \ldots, \mathbf{z}_{N_{\mathrm{T}}, k}^{\mathrm{T}}\right)$.

Each UAV is equipped with a sensor able to observe a subset of the target state space $\mathbb{R}^{n_{\mathrm{z}}}$ and to acquire information on targets belonging to this subset. For a given value $\mathbf{r}_{i, k}$ of the state of UAV $i$, the observed subset is denoted as $\mathbb{F}_{i}\left(\mathbf{r}_{i, k}\right) \subset \mathbb{R}^{n_{\mathrm{z}}}$. One assumes that from the observation of $\mathbb{F}_{i}\left(\mathbf{r}_{i, k}\right)$ at time $k$, UAV $i$ is able to get a list $\mathscr{L}_{i, k}$ of indices of detected targets, i.e.,

$$
j \in \mathscr{L}_{i, k} \Leftrightarrow \mathbf{z}_{j, k} \in \mathbb{F}_{i}\left(\mathbf{r}_{i, k}\right) .
$$

According to (3), the probability of non-detection of a target, as well as the probability of false alarm are null. The processing of $\mathbb{F}_{i}\left(\mathbf{r}_{i, k}\right)$ leads also, for each target $j \in \mathscr{L}_{i, k}$, to a noisy observation of the state $\mathbf{z}_{j, k}$ described as

$$
\mathbf{y}_{i, j, k}=\mathbf{h}_{i}\left(\mathbf{r}_{i, k}, \mathbf{z}_{j, k}\right)+\mathbf{w}_{i, j, k}
$$

where $\mathbf{h}_{i}$ is the observation equation of UAV $i$ and $\mathbf{w}_{i, j, k}$ is the measurement noise bounded in the known box $\left[\mathbf{w}_{k}\right]$.

$\mathbb{I}_{i, k}$ gathers the information available to UAV $i$ at time $k$. From $\mathbb{I}_{i, k}$, UAV $i$ is able to evaluate $\mathscr{D}_{i, k}$, the set of indices of targets already detected or which presence has been signaled by an other UAV of the fleet to UAV $i$. $\mathbb{I}_{i, k}$ is also used to evaluate a set of set estimates $\mathbb{Z}_{i, k}=\left\{\mathbb{Z}_{i, j, k}\right\}_{j \in \mathscr{D}_{i, k}}$ containing the state values of the already detected targets. $\mathbb{Z}_{i, j, k}$ contains all possible values of $\mathbf{z}_{j, k}$ that are consistent with the information available to UAV $i$ at time $k$. Moreover, UAV $i$ also maintains a set $\overline{\mathbb{Z}}_{i, k}$ containing the possible state values of not detected targets.

Consider a measure $\Phi$ of the target state estimation uncertainty. This measure involves the set estimates of the already detected targets as well as $\overline{\mathbb{Z}}_{i, k}$. A possible choice is

$$
\Phi\left(\mathbb{Z}_{i, k}, \overline{\mathbb{Z}}_{i, k}\right)=\frac{1}{\max \left\{1,\left|\mathscr{D}_{i, k}\right|\right\}} \sum_{j \in \mathscr{D}_{i, k}} \phi\left(\mathbb{Z}_{i, j, k}\right)+\alpha \phi\left(\overline{\mathbb{Z}}_{i, k}\right)
$$

where $\phi\left(\mathbb{Z}_{i, j, k}\right)$ is the volume of the set $\mathbb{Z}_{i, j, k},\left|\mathscr{D}_{i, k}\right|$ is the cardinal number of $\mathscr{D}_{i, k}$, and $\alpha$ some parameter to adjust the relative importance of the average state estimation uncertainty of detected targets and of not yet detected targets. The (target state) estimation uncertainty at time $k$ is defined as the average of $\Phi\left(\mathbb{Z}_{i, k}, \overline{\mathbb{Z}}_{i, k}\right)$ computed for all $i=1, \ldots, N_{\mathrm{U}}$

$$
\Phi_{k}=\frac{1}{N_{\mathrm{U}}} \sum_{i=1}^{N_{\mathrm{U}}} \Phi\left(\mathbb{Z}_{i, k}, \overline{\mathbb{Z}}_{i, k}\right)
$$

When two UAVs with indexes $i_{1}$ and $i_{2}$ come in vicinity, they are able to exchange their respective information, which allows each of the UAVs to update their set estimates.

Our aim is to design the sequences of control inputs for each UAV so as to minimize the estimation uncertainty $\Phi_{k}$ for each $k$. For that purpose, one will first consider a single UAV and show the way it may account for additional information at time $k+1$, coming either from its own sensor, or from neighboring UAVs. Then, an evaluation of the control input to apply at time $k$ to each UAV to minimize the estimation uncertainty at time $k+1$ will be described.

\section{Evolution of SET Estimates FOR A GIVEN UAV}

In what follows, we consider the evolution with time of the set estimates $\mathscr{D}_{i, k}, \mathbb{Z}_{i, k}$, and $\overline{\mathbb{Z}}_{i, k}$ managed by a given UAV $i$. These set estimates are evaluated considering a generalization of the nonlinear recursive set-membership state estimator introduced in [9]. As the classical Kalman filter, it alternates prediction and correction steps. For the initialization, at time $t=0$, one has $\mathscr{D}_{i, 0}=\emptyset, \mathbb{Z}_{i, 0}=\emptyset$, and $\overline{\mathbb{Z}}_{i, 0}=\mathbb{Z}_{0}$ for $i=1, \ldots, N_{\mathrm{U}}$.

\section{A. Prediction step}

Assume that at time $k, \mathscr{D}_{i, k}, \mathbb{Z}_{i, k}$, and $\overline{\mathbb{Z}}_{i, k}$ are available to UAV $i$. At time $k+1$, without additional information, the predicted value of $\mathscr{D}_{i, k}$ is $\mathscr{D}_{i, k+1 \mid k}=\mathscr{D}_{i, k}$.

For each target already detected with index $j \in \mathscr{D}_{i, k+1 \mid k}$, UAV $i$ is also able to evaluate the set of possible target state values at time $k+1$, i.e., the set of all target state values that are consistent with $\mathbb{Z}_{i, j, k}$, with the dynamics (2), and the bounded state perturbation

$$
\begin{aligned}
\mathbb{Z}_{i, j, k+1 \mid k} & =\left\{\mathbf{f}_{k}^{Z}(\mathbf{z}, \mathbf{v}) \mid \mathbf{z} \in \mathbb{Z}_{i, j, k}, \mathbf{v} \in\left[\mathbf{v}_{k}\right]\right\} \\
& =\mathbf{f}_{k}^{Z}\left(\mathbb{Z}_{i, j, k},\left[\mathbf{v}_{k}\right]\right) .
\end{aligned}
$$

Similarly, the predicted set $\overline{\mathbb{Z}}_{i, k+1 \mid k}$ has to contain all possible state values of potentially undetected targets. Since all targets have been assumed to evolve according to the same dynamics (2), it is evaluated as follows

$$
\overline{\mathbb{Z}}_{i, k+1 \mid k}=\mathbf{f}_{k}^{\mathrm{Z}}\left(\overline{\mathbb{Z}}_{i, k},\left[\mathbf{v}_{k}\right]\right) \text {. }
$$

\section{B. Correction step from measurements}

Assume that at time $k+1$, UAV $i$ evaluates $\mathscr{L}_{i, k+1}$ from the observation of $\mathbb{F}_{i}\left(\mathbf{r}_{i, k+1}\right)$ and, for each $j \in \mathscr{L}_{i, k+1}$, has access to $\mathbf{y}_{i, j, k+1}$ obtained using (4). Consequently

$$
\mathbb{I}_{i, k+1}=\mathbb{I}_{i, k} \cup\left\{\mathscr{L}_{i, k+1},\left\{\mathbf{y}_{i, j, k+1}\right\}_{j \in \mathscr{L}_{i, k+1}}\right\} \text {. }
$$

Three cases have to be considered.

If $j \in \mathscr{L}_{i, k+1} \cap \mathscr{D}_{i, k+1 \mid k}$, Target $j$, which has already been detected, is observed again at time $k+1$ in $\mathbb{F}_{i}\left(\mathbf{r}_{i, k+1}\right)$. The set estimate of its state at time $k+1$ has to account for the predicted set $\mathbb{Z}_{i, j, k+1 \mid k}$ and for the obtained measurement $\mathbf{y}_{i, j, k+1}$. The set of all values of $\mathbf{z}_{j, k+1}$ that are consistent with $\mathbb{Z}_{i, j, k+1 \mid k}, \mathbf{y}_{i, j, k+1}$, the measurement equation (4), and the measurement noise bound $\left[\mathbf{w}_{k+1}\right]$ is

$$
\begin{aligned}
\mathbb{Z}_{i, j, k+\mid k+1} & =\left\{\mathbf{z} \in \mathbb{Z}_{i, j, k+1 \mid k} \mid \mathbf{h}_{k+1}\left(\mathbf{r}_{i, k+1}, \mathbf{z}\right) \in \mathbf{y}_{i, j, k+1}-\left[\mathbf{w}_{k+1}\right]\right\} \\
& =\mathbb{Z}_{i, j, k+1 \mid k} \cap \mathbf{h}_{i, k+1}^{-1}\left(\mathbf{y}_{i, j, k+1}-\left[\mathbf{w}_{k+1}\right]\right),
\end{aligned}
$$


where $\mathbf{h}_{i, k+1}^{-1}\left(\mathbf{y}_{i, j, k+1}-\left[\mathbf{w}_{k}\right]\right)$ is the pre-image of the box $\mathbf{y}_{i, j, k+1}-\left[\mathbf{w}_{k}\right]$ by the function $\mathbf{h}_{i, k+1}(\mathbf{z})=\mathbf{h}_{k+1}\left(\mathbf{r}_{i, k+1}, \mathbf{z}\right)$.

If $j \in \mathscr{L}_{i, k+1}$ but $j \notin \mathscr{D}_{i, k+1 \mid k}$, a new target has been detected. Before detection, this new target is only known to belong to $\overline{\mathbb{Z}}_{i, k+1 \mid k}$. One has also to take into account the measurement $\mathbf{y}_{i, j, k+1}$ related to this newly detected target. The set of all values of $\mathbf{z}_{j, k+1}$ that are consistent with $\overline{\mathbb{Z}}_{i, k+1 \mid k}$, $\mathbf{y}_{i, j, k+1}$, the measurement equation (4), and the measurement noise bound $\left[\mathbf{w}_{k+1}\right]$ is in this case

$$
\begin{aligned}
\mathbb{Z}_{i, j, k+1 \mid k+1} & =\left\{\mathbf{z} \in \overline{\mathbb{Z}}_{i, k+1 \mid k} \mid \mathbf{h}_{k+1}\left(\mathbf{r}_{i, k+1}, \mathbf{z}\right) \in \mathbf{y}_{i, j, k+1}-\left[\mathbf{w}_{+1}\right]\right\} \\
& =\overline{\mathbb{Z}}_{i, k+1 \mid k} \cap \mathbf{h}_{i, k+1}^{-1}\left(\mathbf{y}_{i, j, k+1}-\left[\mathbf{w}_{k+1}\right]\right) .
\end{aligned}
$$

When $j \notin \mathscr{L}_{i, k+1}$ but $j \in \mathscr{D}_{i, k+1 \mid k}$, Target $j$, which was previously detected is not observed in $\mathbb{F}_{i}\left(\mathbf{r}_{i, k+1}\right)$. The set of all values of $\mathbf{z}_{j, k+1}$ that are consistent with $\mathbb{Z}_{i, j, k+1 \mid k}$, and that do not belong to $\mathbb{F}_{i}\left(\mathbf{r}_{i, k+1}\right)$ is then

$$
\mathbb{Z}_{i, j, k+1 \mid k+1}=\mathbb{Z}_{i, j, k+1 \mid k} \backslash \mathbb{F}_{i}\left(\mathbf{r}_{i, k+1}\right),
$$

where $\mathbb{B} \backslash \mathbb{A}=\{x \in \mathbb{B} \mid x \notin \mathbb{A}\}$.

Two additional update equations have to be considered. First, the set of already detected targets has to incorporate the newly detected targets

$$
\mathscr{D}_{i, k+1 \mid k+1}=\mathscr{D}_{i, k+1 \mid k} \cup \mathscr{L}_{i, k+1} \text {. }
$$

Second, to evaluate the set containing the state of targets still to be detected, one has to account for the fact that all targets in $\mathbb{F}_{i}\left(\mathbf{r}_{i, k+1}\right)$ have been processed. Thus, one has

$$
\overline{\mathbb{Z}}_{i, k+1 \mid k+1}=\overline{\mathbb{Z}}_{i, k+1 \mid k} \backslash \mathbb{F}_{i}\left(\mathbf{r}_{i, k+1}\right) .
$$

At time $k+1$, one has thus $\mathscr{D}_{i, k+1}=\mathscr{D}_{i, k+1 \mid k+1}, \mathbb{Z}_{i, k+1}=$ $\mathbb{Z}_{i, k+1 \mid k+1}$, and $\overline{\mathbb{Z}}_{i, k+1}=\overline{\mathbb{Z}}_{i, k+1 \mid k+1}$.

\section{Correction step from communications}

In this section, we consider the updates performed after a communication between two neighboring UAVs $i_{1}$ and $i_{2}$ occurring in the time interval $] k, k+1[$. Assume that at time $k$, UAV $i$ has access to the sets $\mathscr{D}_{i, k}, \mathbb{Z}_{i, k}$, and $\overline{\mathbb{Z}}_{i, k}$. Each UAV broadcasts a message containing a description of these sets.

Assume in what follows that UAV $i_{1}$ has received a message from UAV $i_{2}$. then $\mathscr{D}_{i_{1}, k+}=\mathscr{D}_{i_{1}, k} \cup \mathscr{D}_{i_{2}, k}$, to account for the targets seen by both UAVs.

The update equation for the set containing the state of a given target depends on whether it has been previously seen by both UAVs $i_{1}$ and $i_{2}$ or by a single UAV only. If $j \in$ $\mathscr{D}_{i_{1}, k} \cap \mathscr{D}_{i_{2}, k}$, both UAVs have already detected Target $j$ and $\mathbb{Z}_{i_{1}, j, k+}=\mathbb{Z}_{i_{1}, j, k} \cap \mathbb{Z}_{i_{2}, j, k}$. If $j \in \mathscr{D}_{i_{1}, k}$ but $j \notin \mathscr{D}_{i_{2}, k}$, UAV $i_{2}$ has not detected Target $j$, but it nevertheless knows that its state is within $\overline{\mathbb{Z}}_{i_{2}, k}$. As a consequence $\mathbb{Z}_{i_{1}, j, k+}=\mathbb{Z}_{i_{1}, j, k} \cap \overline{\mathbb{Z}}_{i_{2}, k}$. Similarly, if $j \notin \mathscr{D}_{i_{1}, k}$ but $j \in \mathscr{D}_{i_{2}, k}, \mathbb{Z}_{i_{1}, j, k+}=\overline{\mathbb{Z}}_{i_{1}, k} \cap \mathbb{Z}_{i_{2}, j, k}$.

Finally, the set containing the state of targets to be detected is updated according to $\overline{\mathbb{Z}}_{i_{1}, k+}=\overline{\mathbb{Z}}_{i_{1}, k} \cap \overline{\mathbb{Z}}_{i_{2}, k}$, since each part of the state space that has been shown by one of the UAVs not to contain a target can be eliminated from the search space for undetected targets.

\section{UAV TRAJECTORY COMPUTATION}

Here, we determine the sequence of control inputs that have to be applied at time $k$ to each UAV so as to minimize the target state estimation uncertainty $\Phi_{k+1}$. One starts considering a single UAV $i$ and $\Phi\left(\mathbb{Z}_{i, k+1}, \overline{\mathbb{Z}}_{i, k+1}\right)$. Then several communicating UAVs will be considered.

\section{A. Control input design for a single UAV}

Assume that at time $k, \mathscr{D}_{i, k}, \mathbb{Z}_{i, k}$, and $\overline{\mathbb{Z}}_{i, k}$ are available to UAV $i$. Using the results of Section III-A, UAV $i$ is able to evaluate $\mathscr{D}_{i, k+1 \mid k}, \mathbb{Z}_{i, k+1 \mid k}$, and $\overline{\mathbb{Z}}_{i, k+1 \mid k}$. One has to determine the impact of a given control input $\mathbf{u}$ applied to UAV $i$ on $\Phi\left(\mathbb{Z}_{i, k+1}, \overline{\mathbb{Z}}_{i, k+1}\right)$. Our aim is to evaluate

$$
\begin{aligned}
\widehat{\mathbf{u}} & =\arg \min _{\mathbf{u} \in \mathbb{U}} \Phi\left(\mathbb{Z}_{i, k+1}, \overline{\mathbb{Z}}_{i, k+1}\right) \\
& =\arg \min _{\mathbf{u} \in \mathbb{U}} \frac{\sum_{j \in \mathscr{D}_{i, k+1}} \phi\left(\mathbb{Z}_{i, j, k+1}\right)+\alpha \phi\left(\overline{\mathbb{Z}}_{i, k+1}\right)}{\max \left\{1,\left|\mathscr{D}_{i, k+1}\right|\right\}} .
\end{aligned}
$$

1) Impact of the choice $\mathbf{u}_{i}=\mathbf{u} \in \mathbb{U}$ : Four situations have to be considered for a given value $\mathbf{u}_{i}=\mathbf{u} \in \mathbb{U}$.

The first situation is for targets with indices $j \in \mathscr{L}_{i, k+1} \cap$ $\mathscr{D}_{i, k+1 \mid k}$. When applying the control input $\mathbf{u}_{i}=\mathbf{u} \in \mathbb{U}$, using (1), one gets $\mathbf{r}_{i, k+1}=\mathbf{f}_{k}^{\mathrm{R}}\left(\mathbf{r}_{i, k}, \mathbf{u}\right)$. Using (10), the updated estimation uncertainty for that target will be

$$
\begin{aligned}
\phi^{11}\left(\mathbb{Z}_{i, j, k+1}\right) & =\phi\left(\left\{\mathbf{z} \in \mathbb{Z}_{i, j, k+1 \mid k} \mid\right.\right. \\
& \left.\left.\mathbf{h}_{k+1}\left(\mathbf{r}_{i, k+1}, \mathbf{z}\right) \in \mathbf{y}_{i, j, k+1}-\left[\mathbf{w}_{k+1}\right]\right\}\right) .
\end{aligned}
$$

Here and in what follows, $\phi^{\delta_{k} \delta_{k+1}}$ indicates whether the considered target has been detected at time $k\left(\delta_{k}=1\right)$ or not $\left(\delta_{k}=0\right)$ and whether it is detected at time $k+1\left(\delta_{k+1}=1\right)$ or not $\left(\delta_{k+1}=0\right)$. The measurement $\mathbf{y}_{i, j, k+1}$ depends on $\mathbf{r}_{i, k+1}$, on the actual value of the target state $\mathbf{z}_{j, k+1}$ at time $k+1$, and on the realization of the measurement noise $\mathbf{w}_{i, j, k}$. Consequently, one may rewrite (16) as

$$
\begin{array}{r}
\phi^{11}\left(\mathbb{Z}_{i, j, k+1}\right)=\phi\left(\left\{\mathbf{z} \in \mathbb{Z}_{i, j, k+1 \mid k} \mid \mathbf{h}_{k+1}\left(\mathbf{f}_{k}^{\mathrm{R}}\left(\mathbf{r}_{i, k}, \mathbf{u}\right), \mathbf{z}\right) \in\right.\right. \\
\left.\left.\mathbf{h}_{k+1}\left(\mathbf{f}_{k}^{\mathrm{R}}\left(\mathbf{r}_{i, k}, \mathbf{u}\right), \mathbf{z}_{j, k+1}\right)+\mathbf{w}_{i, j, k+1}-\left[\mathbf{w}_{k+1}\right]\right\}\right) .
\end{array}
$$

In (17), when the choice $\mathbf{u}_{i}=\mathbf{u}$ is made at time $k, \mathbf{w}_{i, j, k+1}$ is only known to belong to $\left[\mathbf{w}_{k+1}\right]$ and $\mathbf{z}_{j, k+1}$ is only known to belong to $\mathbb{Z}_{i, j, k+1 \mid k}$ and to $\mathbb{F}_{i}\left(\mathbf{f}_{k}^{\mathrm{R}}\left(\mathbf{r}_{i, k}, \mathbf{u}\right)\right)$, since Target $j$ is assumed to be detected at time $k+1$. The future corrected set estimate $\mathbb{Z}_{i, j, k+1}$ is relatively difficult to evaluate considering only the previous bounds on the noises and the fact that $\mathbf{z}_{j, k+1} \in \mathbb{Z}_{i, j, k+1 \mid k} \cap \mathbb{F}_{i}\left(\mathbf{f}_{k}^{\mathrm{R}}\left(\mathbf{r}_{i, k}, \mathbf{u}\right)\right)$.

The second situation corresponds to $j \in \mathscr{L}_{i, k+1}$ but $j \notin$ $\mathscr{D}_{i, k+1 \mid k}$, i.e., a new target is detected. Using (11), one gets

$$
\begin{aligned}
\phi^{01}\left(\mathbb{Z}_{i, j, k+1}\right) & =\phi\left(\left\{\mathbf{z} \in \overline{\mathbb{Z}}_{i, k+1 \mid k} \mid\right.\right. \\
& \left.\left.\mathbf{h}_{k+1}\left(\mathbf{r}_{i, k+1}, \mathbf{z}\right) \in \mathbf{y}_{i, j, k+1}-\left[\mathbf{w}_{k+1}\right]\right\}\right) .
\end{aligned}
$$

The third situation corresponds to $j \notin \mathscr{L}_{i, k+1}$ but $j \in$ $\mathscr{D}_{i, k+1 \mid k}$, i.e., a previously detected target is no more detected. Using (12) and (1), one gets

$$
\phi^{10}\left(\mathbb{Z}_{i, j, k+1}\right)=\phi\left(\mathbb{Z}_{i, j, k+1 \mid k} \backslash \mathbb{F}_{i}\left(\mathbf{f}_{k}^{\mathrm{R}}\left(\mathbf{r}_{i, k}, \mathbf{u}\right)\right)\right) .
$$


The last situation corresponds to undetected targets for which one has to evaluate $\phi\left(\overline{\mathbb{Z}}_{i, k+1}\right)$. Using (14) and (1), one gets

$$
\phi\left(\overline{\mathbb{Z}}_{i, k+1}\right)=\phi\left(\overline{\mathbb{Z}}_{i, k+1 \mid k} \backslash \mathbb{F}_{i}\left(\mathbf{f}_{k}^{\mathrm{R}}\left(\mathbf{r}_{i, k}, \mathbf{u}\right)\right)\right) .
$$

2) Bounding $\Phi\left(\mathbb{Z}_{i, k+1}, \overline{\mathbb{Z}}_{i, k+1}\right)$ : Now, to evaluate (15) when $\mathbf{u}_{i}=\mathbf{u}$, one has to isolate the contributions of the potentially newly detected targets. To simplify notations, one assumes that $\left|\mathscr{D}_{i, k}\right| \geqslant 1$ and gets

$$
\begin{aligned}
& \frac{1}{\left|\mathscr{D}_{i, k+1}\right|} \sum_{j \in \mathscr{D}_{i, k+1}} \phi\left(\mathbb{Z}_{i, j, k+1}\right) \\
& =\frac{1}{\left|\mathscr{D}_{i, k+1}\right|}\left(\sum_{j \in \mathscr{D}_{i, k}} \phi\left(\mathbb{Z}_{i, j, k+1}\right)+\sum_{j \in \mathscr{D}_{i, k+1} \backslash \mathscr{D}_{i, k}} \phi^{01}\left(\mathbb{Z}_{i, j, k+1}\right)\right) .
\end{aligned}
$$

The term $\phi^{01}\left(\mathbb{Z}_{i, j, k+1}\right)$ in (21) is difficult to evaluate accurately, but it may be upper bounded using (18) as follows

$$
\begin{aligned}
& \phi^{01}\left(\mathbb{Z}_{i, j, k+1}\right)=\max _{\mathbf{w} \in\left[\mathbf{w}_{k}\right] \mathbf{z}^{\prime} \in \overline{\mathbb{Z}}_{i, k+1 \mid k} \cap \max _{i}\left(\mathbf{f}_{k}^{\mathrm{R}}\left(\mathbf{r}_{i, k}, \mathbf{u}\right)\right)} \\
& \phi\left(\left\{\mathbf{z} \in \overline{\mathbb{Z}}_{i, k+1 \mid k} \mid \mathbf{h}_{k+1}\left(\mathbf{f}_{k}^{\mathrm{R}}\left(\mathbf{r}_{i, k}, \mathbf{u}\right), \mathbf{z}\right) \in\right.\right. \\
& \left.\left.\mathbf{h}_{k+1}\left(\mathbf{f}_{k}^{\mathrm{R}}\left(\mathbf{r}_{i, k}, \mathbf{u}\right), \mathbf{z}^{\prime}\right)+\mathbf{w}-\left[\mathbf{w}_{k+1}\right]\right\}\right) .
\end{aligned}
$$

This upper bound is independent of the index $j$ of the newly detected target. Then

$$
\phi^{01}\left(\mathbb{Z}_{i, j, k+1}\right) \leqslant \bar{\phi}^{01}\left(\overline{\mathbb{Z}}_{i, k+1 \mid k}\right),
$$

with $\bar{\phi}^{01}\left(\overline{\mathbb{Z}}_{i, k+1 \mid k}\right)=\phi\left(\overline{\mathbb{Z}}_{i, k+1 \mid k} \cap \mathbb{F}_{i}\left(\mathbf{f}_{k}^{\mathrm{R}}\left(\mathbf{r}_{i, k}, \mathbf{u}\right)\right)\right)$.

Using (22), one may upper bound (21) as

$$
\begin{aligned}
& \frac{1}{\left|\mathscr{D}_{i, k+1}\right|} \sum_{j \in \mathscr{D}_{i, k+1}} \phi\left(\mathbb{Z}_{i, j, k+1}\right) \\
& \leqslant \frac{1}{\left|\mathscr{D}_{i, k+1}\right|}\left(\sum_{j \in \mathscr{D}_{i, k}} \phi\left(\mathbb{Z}_{i, j, k+1}\right)+\sum_{j \in \mathscr{D}_{i, k+1} \backslash \mathscr{D}_{i, k}} \bar{\phi}^{01}\left(\overline{\mathbb{Z}}_{i, k+1 \mid k}\right)\right) \\
& \leqslant \frac{1}{\left|\mathscr{D}_{i, k+1}\right|} \sum_{j \in \mathscr{D}_{i, k}} \phi\left(\mathbb{Z}_{i, j, k+1}\right)+\frac{\left|\mathscr{D}_{i, k+1}\right|-\left|\mathscr{D}_{i, k}\right|}{\left|\mathscr{D}_{i, k+1}\right|} \bar{\phi}^{01}\left(\overline{\mathbb{Z}}_{i, k+1 \mid k}\right) \\
& \leqslant \frac{1}{\left|\mathscr{D}_{i, k+1}\right|} \sum_{j \in \mathscr{D}_{i, k}} \phi\left(\mathbb{Z}_{i, j, k+1}\right)+\bar{\phi}^{01}\left(\overline{\mathbb{Z}}_{i, k+1 \mid k}\right) .
\end{aligned}
$$

Now, to upper bound $\phi\left(\mathbb{Z}_{i, j, k+1}\right)$, one has to consider whether $j \in \mathscr{L}_{i, k+1}$ to get

$$
\phi\left(\mathbb{Z}_{i, j, k+1}\right) \leqslant \max \left\{\phi^{10}\left(\mathbb{Z}_{i, j, k+1}\right), \phi^{11}\left(\mathbb{Z}_{i, j, k+1}\right)\right\} .
$$

Since $\mathbb{Z}_{i, j, k+1 \mid k} \backslash \mathbb{F}_{i}\left(\mathbf{f}_{k}^{\mathrm{R}}\left(\mathbf{r}_{i, k}, \mathbf{u}\right)\right)$ in (19) is easily obtained using the SIVIA algorithm [10], to get $\phi^{10}\left(\mathbb{Z}_{i, j, k+1}\right)$, it remains to evaluate $\phi^{11}\left(\mathbb{Z}_{i, j, k+1}\right)$. As for $\phi^{01}\left(\mathbb{Z}_{i, j, k+1}\right)$, an upper bound $\bar{\phi}^{11}\left(\mathbb{Z}_{i, j, k+1}\right)$ will be evaluated from (17), using a similar approach to get

$$
\begin{aligned}
\phi^{11}\left(\mathbb{Z}_{i, j, k+1}\right)= & \max _{\mathbf{w} \in\left[\mathbf{w}_{k}\right] \mathbf{z}^{\prime} \in \mathbb{Z}_{i, j, k+1 \mid k} \cap \mathbb{F}_{i}\left(\mathbf{f}_{k}^{\mathrm{R}}\left(\mathbf{r}_{i, k}, \mathbf{u}\right)\right)} \\
& \phi\left(\left\{\mathbf{z} \in \mathbb{Z}_{i, j, k+1 \mid k} \mid \mathbf{h}_{k+1}\left(\mathbf{f}_{k}^{\mathrm{R}}\left(\mathbf{r}_{i, k}, \mathbf{u}\right), \mathbf{z}\right) \in\right.\right. \\
& \left.\left.\mathbf{h}_{k+1}\left(\mathbf{f}_{k}^{\mathrm{R}}\left(\mathbf{r}_{i, k}, \mathbf{u}\right), \mathbf{z}^{\prime}\right)+\mathbf{w}-\left[\mathbf{w}_{k+1}\right]\right\}\right),
\end{aligned}
$$

which can be further upper bounded as

$$
\phi^{11}\left(\mathbb{Z}_{i, j, k+1}\right) \leqslant \bar{\phi}^{11}\left(\mathbb{Z}_{i, j, k+1}\right),
$$

with $\bar{\phi}^{11}\left(\mathbb{Z}_{i, j, k+1}\right)=\phi\left(\mathbb{Z}_{i, j, k+1 \mid k} \cap \mathbb{F}_{i}\left(\mathbf{f}_{k}^{\mathrm{R}}\left(\mathbf{r}_{i, k}, \mathbf{u}\right)\right)\right)$.

Finally, using (20), (23), (24), and (25) in (15), one obtains

$$
\begin{aligned}
\Phi\left(\mathbb{Z}_{i, k+1}, \overline{\mathbb{Z}}_{i, k+1}\right) \leqslant \frac{1}{\left|\mathscr{D}_{i, k+1}\right|} \sum_{j \in \mathscr{D}_{i, k}} \max \left\{\phi^{10}\left(\mathbb{Z}_{i, j, k+1}\right),\right. \\
\left.\bar{\phi}^{11}\left(\mathbb{Z}_{i, j, k+1}\right)\right\}+\bar{\phi}^{01}\left(\overline{\mathbb{Z}}_{i, k+1 \mid k}\right)+\alpha \phi\left(\overline{\mathbb{Z}}_{i, k+1}\right) .
\end{aligned}
$$

Since $\left|\mathscr{D}_{i, k+1}\right|$ is unknown at time $k$, but $\left|\mathscr{D}_{i, k}\right| \leqslant\left|\mathscr{D}_{i, k+1}\right|$, one may further upper bound $\Phi\left(\mathbb{Z}_{i, k+1}, \overline{\mathbb{Z}}_{i, k+1}\right)$ as

$$
\begin{aligned}
\Phi\left(\mathbb{Z}_{i, k+1}, \overline{\mathbb{Z}}_{i, k+1}\right) \leqslant \frac{1}{\left|\mathscr{D}_{i, k}\right|} \sum_{j \in \mathscr{D}_{i, k}} \max \left\{\phi^{10}\left(\mathbb{Z}_{i, j, k+1}\right),\right. \\
\left.\bar{\phi}^{11}\left(\mathbb{Z}_{i, j, k+1}\right)\right\}+\bar{\phi}^{01}\left(\overline{\mathbb{Z}}_{i, k+1 \mid k}\right)+\alpha \phi\left(\overline{\mathbb{Z}}_{i, k+1}\right),
\end{aligned}
$$

which is independent of the number of newly detected targets.

In (26), to evaluate

$$
\phi\left(\overline{\mathbb{Z}}_{i, k+1}\right)=\phi\left(\overline{\mathbb{Z}}_{i, k+1 \mid k} \backslash \mathbb{F}_{i}\left(\mathbf{f}_{k}^{\mathrm{R}}\left(\mathbf{r}_{i, k}, \mathbf{u}\right)\right)\right)
$$

one has to compute the difference between two sets. An outer-approximation of this set difference is again evaluated, e.g., using SIVIA, [10].

When $\overline{\mathbb{Z}}_{i, k}$ is large, i.e., when a large part of the state space is unexplored, the chosen control input is likely to be such that $\phi\left(\overline{\mathbb{Z}}_{i, k+1}\right)$ is minimized. When the uncertainty on the state of already detected targets increases, the chosen control input will correspond to a trade-off between $\phi^{10}\left(\mathbb{Z}_{i, j, k+1}\right)$ and $\phi\left(\overline{\mathbb{Z}}_{i, k+1}\right)$, as expected. The value of $\alpha$ helps finding a trade-off between the search for not already detected targets and the reduction of the estimation uncertainty for already detected targets.

\section{B. Control input design for several cooperating UAVs}

In this section, one assumes that all UAVs communicate their set estimates at each time instant $k$ once they have taken into account their own measurements. They also send their current state vector. As a consequence, all UAVs share the same information, and the set estimates are equal. In this context, the control input design for all UAVs may be performed by each UAV independently, while accounting for the presence of other UAVs. Alternatively, a leader UAV may evaluate the control inputs of all UAVs and broadcast these inputs at the beginning of each time interval.

The evaluation of the one-step control inputs to apply at time $k$ may be formulated as

$$
\left(\widehat{\mathbf{u}}_{1}, \ldots, \widehat{\mathbf{u}}_{N_{\mathrm{U}}}\right)=\arg \underset{\left(\mathbf{u}_{1}, \ldots, \mathbf{u}_{N_{\mathrm{U}}}\right) \in \mathbb{U}^{N_{\mathrm{U}}}}{\min } \frac{1}{N_{\mathrm{U}}} \sum_{i=1}^{N_{\mathrm{U}}} \Phi\left(\mathbb{Z}_{i, k+1}, \overline{\mathbb{Z}}_{i, k+1}\right) .
$$

The $\Phi\left(\mathbb{Z}_{i, k}, \overline{\mathbb{Z}}_{i, k}\right) \mathrm{s}$ are all equal, since they are evaluated after each correction step from communications. Without loss of generality, one can focus on $\Phi\left(\mathbb{Z}_{1, k}, \overline{\mathbb{Z}}_{1, k}\right)$. To solve 
(28), one has again to evaluate the impact of $\mathbf{u}_{1}, \ldots, \mathbf{u}_{N_{\mathrm{U}}}$ on $\Phi\left(\mathbb{Z}_{1, k+1}, \overline{\mathbb{Z}}_{1, k+1}\right)$.

First, for a given choice $\left(\mathbf{u}_{1}, \ldots, \mathbf{u}_{N_{\mathrm{U}}}\right) \in \mathbb{U}^{N_{\mathrm{U}}}$ of the control inputs, $\mathscr{D}_{1, k \mid k}$ will be updated as

$$
\mathscr{D}_{1, k+1 \mid k+1}=\mathscr{D}_{1, k \mid k} \cup \bigcup_{i=1}^{N_{\mathrm{U}}} \mathscr{L}_{i, k+1}
$$

where the terms $\mathscr{L}_{i, k+1}$ depend on the relative configuration of the UAVs with respect to the undetected targets.

The set containing the state of potentially undetected targets is then easily deduced from (14)

$$
\overline{\mathbb{Z}}_{1, k+1 \mid k+1}=\overline{\mathbb{Z}}_{1, k+1 \mid k} \backslash \bigcup_{i=1}^{N_{\mathrm{U}}} \mathbb{F}_{i}\left(\mathbf{f}_{k}^{\mathrm{R}}\left(\mathbf{r}_{i, k}, \mathbf{u}_{i}\right)\right) .
$$

As a consequence

$$
\phi\left(\overline{\mathbb{Z}}_{1, k+1 \mid k+1}\right)=\phi\left(\overline{\mathbb{Z}}_{1, k+1 \mid k} \backslash \bigcup_{i=1}^{N_{\mathrm{U}}} \mathbb{F}_{i}\left(\mathbf{f}_{k}^{\mathrm{R}}\left(\mathbf{r}_{i, k}, \mathbf{u}_{i}\right)\right)\right) .
$$

To evaluate the components of $\mathbb{Z}_{1, k+1}$, for all $j \in$ $\mathscr{D}_{1, k+1 \mid k+1}$, let $\mathscr{U}_{j, k+1}$ be the set of all UAVs that detect Target $j$ at time $k+1$. One has

$$
i \in \mathscr{U}_{j, k+1} \Leftrightarrow j \in \mathscr{L}_{i, k+1} \text {. }
$$

Moreover, let $\overline{\mathscr{U}}_{j, k+1}=\left\{1, \ldots, N_{\mathrm{U}}\right\} \backslash \mathscr{U}_{j, k+1}$. With these notations, combining (10) and (12), one gets for all $j \in \mathscr{D}_{1, k \mid k}$

$$
\begin{aligned}
\mathbb{Z}_{1, j, k+1} & =\bigcap_{i \in \mathscr{U}_{j, k+1}}\left\{\mathbf{z} \in \mathbb{Z}_{1, j, k+1 \mid k} \mid\right. \\
& \left.\mathbf{h}_{k+1}\left(\mathbf{f}_{k}^{\mathrm{R}}\left(\mathbf{r}_{i, k}, \mathbf{u}_{i}\right), \mathbf{z}\right) \in \mathbf{y}_{i, j, k+1}-\left[\mathbf{w}_{k+1}\right]\right\} \\
& \backslash \bigcup_{i \in \overline{\mathscr{U}}_{j, k+1}} \mathbb{F}_{i}\left(\mathbf{f}_{k}^{\mathrm{R}}\left(\mathbf{r}_{i, k}, \mathbf{u}_{i}\right)\right) .
\end{aligned}
$$

If $\mathscr{U}_{j, k+1}=\emptyset, \mathbb{Z}_{1, j, k+1}$ boils down to

$$
\mathbb{Z}_{1, j, k+1}=\mathbb{Z}_{1, j, k+1 \mid k} \backslash \bigcup_{i=1}^{N_{\mathrm{U}}} \mathbb{F}_{i}\left(\mathbf{f}_{k}^{\mathrm{R}}\left(\mathbf{r}_{i, k}, \mathbf{u}_{i}\right)\right)
$$

leading to

$$
\phi^{10}\left(\mathbb{Z}_{1, j, k+1}\right)=\phi\left(\mathbb{Z}_{1, j, k+1 \mid k} \backslash \bigcup_{i=1}^{N_{\mathrm{U}}} \mathbb{F}_{i}\left(\mathbf{f}_{k}^{\mathrm{R}}\left(\mathbf{r}_{i, k}, \mathbf{u}_{i}\right)\right)\right) .
$$

If $\mathscr{U}_{j, k+1} \neq \emptyset$, consider $i_{1} \in \mathscr{U}_{j, k+1}$, then one has

$$
\begin{aligned}
\mathbb{Z}_{1, j, k+1} & \subset\left\{\mathbf{z} \in \mathbb{Z}_{1, j, k+1 \mid k} \mid\right. \\
& \left.\mathbf{h}_{k+1}\left(\mathbf{f}_{k}^{\mathrm{R}}\left(\mathbf{r}_{i_{1}, k}, \mathbf{u}_{i_{1}}\right), \mathbf{z}\right) \in \mathbf{y}_{i_{1}, j, k+1}-\left[\mathbf{w}_{k+1}\right]\right\},
\end{aligned}
$$

and consequently, in this case

$$
\begin{aligned}
\phi^{11}\left(\mathbb{Z}_{1, j, k+1}\right) \leqslant & \phi\left(\left\{\mathbf{z} \in \mathbb{Z}_{1, j, k+1 \mid k} \mid\right.\right. \\
& \left.\left.\mathbf{h}_{k+1}\left(\mathbf{f}_{k}^{\mathrm{R}}\left(\mathbf{r}_{i_{1}, k}, \mathbf{u}_{i_{1}}\right), \mathbf{z}\right) \in \mathbf{y}_{i_{1}, j, k+1}-\left[\mathbf{w}_{k+1}\right]\right\}\right),
\end{aligned}
$$

where the right hand side term of the inequality corresponds to $\bar{\phi}^{11}\left(\mathbb{Z}_{1, j, k+1}\right)$.
Finally, combining (11) and (14), for all $j \in \mathscr{D}_{1, k+1 \mid k+1}$ with $j \notin \mathscr{D}_{1, k \mid k}$, one has

$$
\begin{aligned}
\mathbb{Z}_{1, j, k+1} & =\bigcap_{i \in \mathscr{U}_{j, k+1}}\left\{\mathbf{z} \in \overline{\mathbb{Z}}_{1, k+1 \mid k} \mid\right. \\
& \left.\mathbf{h}_{k+1}\left(\mathbf{f}_{k}^{\mathrm{R}}\left(\mathbf{r}_{i, k}, \mathbf{u}_{i}\right), \mathbf{z}\right) \in \mathbf{y}_{i, j, k+1}-\left[\mathbf{w}_{k+1}\right]\right\} \\
& \backslash \bigcup_{i \in \overline{\mathscr{U}}_{j, k+1}} \mathbb{F}_{i}\left(\mathbf{f}_{k}^{\mathrm{R}}\left(\mathbf{r}_{i, k}, \mathbf{u}_{i}\right)\right) .
\end{aligned}
$$

In this case, since $j \in \mathscr{D}_{1, k+1 \mid k+1}$ and $j \notin \mathscr{D}_{1, k \mid k}, \mathscr{U}_{j, k+1} \neq \emptyset$. Considering $i_{1} \in \mathscr{U}_{j, k+1}$, one has

$$
\begin{aligned}
\mathbb{Z}_{1, j, k+1} & \subset\left\{\mathbf{z} \in \overline{\mathbb{Z}}_{1, k+1 \mid k} \mid\right. \\
& \left.\mathbf{h}_{k+1}\left(\mathbf{f}_{k}^{\mathrm{R}}\left(\mathbf{r}_{i_{1}, k}, \mathbf{u}_{i_{1}}\right), \mathbf{z}\right) \in \mathbf{y}_{i_{1}, j, k+1}-\left[\mathbf{w}_{k}\right]\right\},
\end{aligned}
$$

and now

$$
\begin{aligned}
\phi^{01}\left(\mathbb{Z}_{1, j, k+1}\right) & \leqslant \phi\left(\left\{\mathbf{z} \in \overline{\mathbb{Z}}_{1, k+1 \mid k} \mid\right.\right. \\
& \left.\left.\mathbf{h}_{k+1}\left(\mathbf{f}_{k}^{\mathrm{R}}\left(\mathbf{r}_{i_{1}, k}, \mathbf{u}_{i_{1}}\right), \mathbf{z}\right) \in \mathbf{y}_{i_{1}, j, k+1}-\left[\mathbf{w}_{k}\right]\right\}\right) \\
& \leqslant \bar{\phi}_{i, k+1}^{01} .
\end{aligned}
$$

As for the control of a single UAV, all these sets depend on the actual target location, which is only known to belong to some set, to the realizations of the measurement noise, and to the realization of the state perturbations. The same upper bound for $\Phi\left(\mathbb{Z}_{1, k+1}, \overline{\mathbb{Z}}_{1, k+1}\right)$ as (26) can be derived here and one resorts to the minimization of this upper bound to design the optimal control inputs.

\section{Simulation RESUlts}

\section{A. Implementation issues}

To implement the proposed approach, one has to evaluate the direct image of a set by a function as in (7) or (8), the reciprocal image of a set by a function, as in (10) and (11), to compute the difference between two sets, as in (13) or (14), and finally, to compute the volume of sets. Here, all sets are described using subpavings, i.e., unions of non-overlapping interval vectors, as in [10] and [9]. The ImageSp algorithm can then be employed to evaluate an outer-approximating subpaving of (7) or (8). The SIVIA algorithm can provide an outer-approximation of the sets defined by (10) and (11). When the observed area is defined by inequalities, the set differences (13) or (14) may also be evaluated using SIVIA, see [10]. Finally, the volume of all these sets is the sum of the volume of the interval vectors forming the approximating subpaving.

The resulting set estimates are guaranteed to contain the actual state, provided that the hypotheses on the noise bounds are satisfied and provided that the measurement process actually satisfies (3).

\section{B. Simulation conditions}

A Matlab simulation has been carried out to illustrate the properties of the proposed approaches. ImageSp and SIVIA have been implemented using the Intlab library [11].

The search of three targets by two cooperating UAVs is considered. The targets are assumed to be static and located in a plane, $\mathbf{z}_{j, k} \in \mathbb{R}^{2}, j=1, \ldots, 3$. The initial search area is a 
square of $500 \times 500 \mathrm{~m}^{2}$. The $\Phi$ function is also expressed in square meters with $\alpha=1$. The UAVs fly at a constant height of $75 \mathrm{~m}$ above the terrain and are equipped with an optical sensor able to detect targets within its field of view defined by a $\left[-\frac{\pi}{8} ; \frac{\pi}{8}\right]$ angle in both azimuth and elevation. The field of view is thus a cone with square basis. When a target is detected at time $k$, one assumes that the measurement equation provides its actual location with an uncertainty bounded in $[-5 \mathrm{~m}, 5 \mathrm{~m}]$ for both components of $\mathbf{z}_{j, k}$.

The UAVs are flying at constant speed of $15 \mathrm{~m} / \mathrm{s}$. The sampling period is $T=1 \mathrm{~s}$. The set $\mathbb{U}$ of admissible control inputs of the UAVs contains the potential speed orientation variation and consists of 20 regularly-spaced valued between $-\pi / 2$ and $\pi / 2$. Both UAVs are able to communicate at each time instant. Their estimates are thus equal, but their control inputs are evaluated in a totally decentralized way, independently for each UAV.

Figure 1 (left) shows the initial locations of the targets (red dots) and of the UAVs and the subpaving obtained when a single measurement has been performed by both UAVs. All displayed boxes represent an outer-approximation of the sets $\overline{\mathbb{Z}}_{1,1}$ or $\overline{\mathbb{Z}}_{2,1}$. The other sets are empty. In Figure 1 (right), one
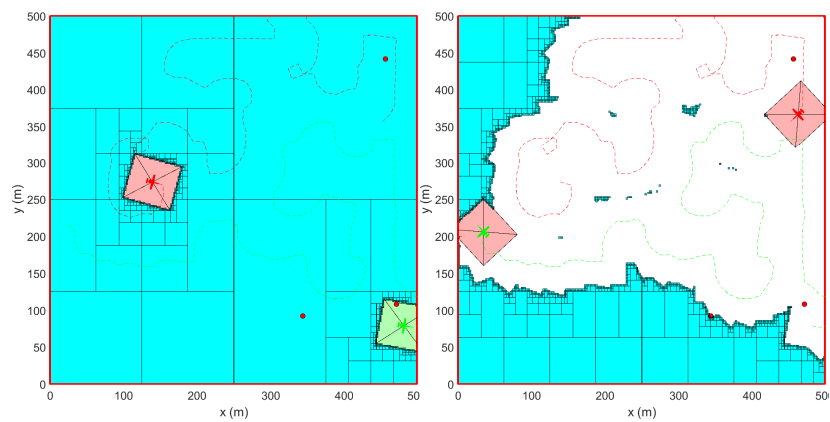

Fig. 1. Initial locations of the UAVs with their sensed area and unknown target locations in red; the boxes form an outer-approximations of the sets $\overline{\mathbb{Z}}_{1,1}$ or $\overline{\mathbb{Z}}_{2,1}$ (left); Final subpaving describing $\overline{\mathbb{Z}}_{1,1}$ or $\overline{\mathbb{Z}}_{2,1}$ after detection of the three targets (in green) by the UAVs (right)

sees that the three targets have been successfully detected. Nevertheless, there may be more target to be detected. The exploration may continue until $\overline{\mathbb{Z}}_{1, k}$ or $\overline{\mathbb{Z}}_{2, k}$ is empty. One can then conclude that all targets have been detected.

Figure 2 presents the evolution with time of $\Phi\left(\mathbb{Z}_{1, k}, \overline{\mathbb{Z}}_{1, k}\right)$, which describes the average target state estimation uncertainty. The control inputs applied to the UAVs are mainly chosen here so as to reduce $\bar{\phi}\left(\overline{\mathbb{Z}}_{i, k+1}\right)$. When a target has been located, the associated estimation uncertainty decreases to less than $100 \mathrm{~m}^{2}$, which corresponds to the volume of the measurement uncertainty box. As soon as a target has been detected, the UAV moves away from it to further decrease the size of $\overline{\mathbb{Z}}_{i, k+1}$.

Videos describing the evolution of the sets $\overline{\mathbb{Z}}_{i, k}$ considering static and moving targets are available at https://drive.google.com/drive/folders/ 1vG9M2xFvJ1z0yU79pfsOs 7 J4 40 ez 6HZM

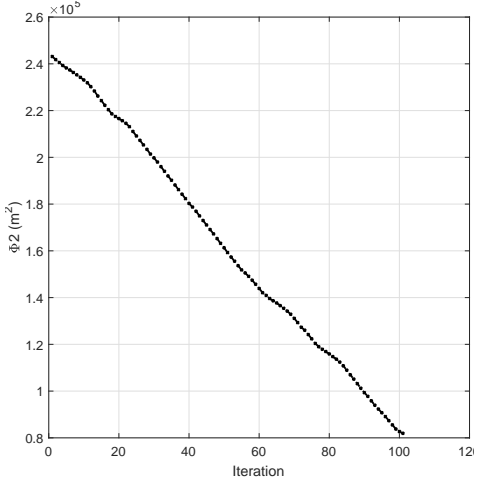

Fig. 2. Evolution of $\Phi\left(\mathbb{Z}_{1, k}, \overline{\mathbb{Z}}_{1, k}\right)$ corresponding to the average target state estimation uncertainty

\section{CONCLUSIONS AND PERSPECTIVES}

This paper considers the search and tracking problem of an unknown number of targets using a fleet of UAVs in a bounded-error context. Set-membership estimates are used to describe the state estimation uncertainty of detected targets. A set containing all state values of targets remaining to be detected is also provided. Both absence or presence of targets in the field of view of UAVs are efficiently taken into account in this set-membership framework. Considering the volume of all these sets at a given time step as a measure of the average estimation uncertainty, one has designed the control inputs minimizing an upper bound of the estimation uncertainty at the next time step.

\section{REFERENCES}

[1] C. Robin and S. Lacroix, "Multi-robot target detection and tracking: taxonomy and survey," Auton. Robots, vol. 40, no. 4, pp. 729-760, 2015.

[2] A. Khan, B. Rinner, and A. Cavallaro, "Cooperative robots to observe moving targets," IEEE trans. Cybern., vol. 48, no. 1, pp. 187-198, 2018.

[3] E. Adamey, A. E. Oğuz, and Ü. Özgüner, "Collaborative multi-msa multi-target tracking and surveillance: a divide \& conquer method using region allocation trees," Jnl Intel. \& Robotic Systems, vol. 87, no. 3-4, pp. 471-485, 2017.

[4] P. Dames, "Distributed multi-target search and tracking using the phd filter," in Proc IEEE Int. Symp. on Multi-Robot and Multi-Agent Systems, 2017, pp. 1-8.

[5] H. Yu, K. Meier, M. Argyle, and R. W. Beard, "Cooperative path planning for target tracking in urban environments using unmanned air and ground vehicles," IEEE/ASME trans. Mechatronics, vol. 20, no. 2, pp. 541-552, 2015.

[6] J. Capitan, M. T. Spaan, L. Merino, and A. Ollero, "Decentralized multi-robot cooperation with auctioned pomdps," Int. Jnl Robotics Research, vol. 32, no. 6, pp. 650-671, 2013.

[7] F. Morbidi and G. L. Mariottini, "Active target tracking and cooperative localization for teams of aerial vehicles," IEEE trans. Control systems technology, vol. 21, no. 5, pp. 1694-1707, 2013.

[8] F. Gu, Y. He, and J. Han, "Active persistent localization of a threedimensional moving target under set-membership uncertainty description through cooperation of multiple mobile robots," IEEE Trans. Industrial Electronics, vol. 62, no. 8, pp. 4958-4971, 2015.

[9] M. Kieffer, L. Jaulin, and E. Walter, "Guaranteed recursive nonlinear state bounding using interval analysis," Int. Jnl Adaptive Control and Signal Proc., vol. 6, no. 3, pp. 193-218, 2002.

[10] L. Jaulin, M. Kieffer, O. Didrit, and E. Walter, Applied Interval Analysis. London: Springer-Verlag, 2001.

[11] S. M. Rump, "INTLAB - INTerval LABoratory," in Developments in Reliable Computing, T. Csendes, Ed. Dordrecht, the Netherlands: Kluwer, 1999, pp. 77-104. 